\title{
Highly efficient base editing in human tripronuclear zygotes
}

\section{Dear Editor,}

There are hundreds of disease-causing single-gene mutations, mainly caused by single-nucleotide substitutions or point mutations rather than small insertions/deletions (indels), and often there are no cures for these diseases. By introducing the CRISPR/Cas9 system into mouse zygotes, disease-causing mutations could be corrected, leading to the production of healthy adult animals (Long et al., 2014; Wang et al., 2013; Wu et al., 2013; Yang et al., 2013). Several studies have demonstrated that CRISPR/Cas9-mediated gene editing could also introduce precise genetic modifications in early human embryos (Kang et al., 2016; Liang et al., 2015; Tang et al., 2017). However, indels rather than singlenucleotide substitutions are obtained frequently, because most DNA double-strand breaks (DSBs) produced by programmable nucleases are repaired by error-prone non-homologous end-joining (NHEJ) rather than homologous recombination (HR) using a template donor DNA. Recently, base editors, composed of cytidine deaminase, Cas9 nickase (nCas9), and uracil DNA glycosylase inhibitor (UGI), have recently been developed to substitute a $C$ at a target site with $\mathrm{T}$ without generating DSBs in plant, yeast, mouse zygotes, and human cells, and shown to be $>100$-fold more efficient than HR at generating point mutations (Kim et al., 2017a; Komor et al., 2016; Liang et al., 2017; Ma et al., 2016; Nishida et al., 2016; Zong et al., 2017). Moreover, the genome-targeting scope has been increased by using staphylococcus aureus CRISPR/Cas9 (SaCas9) with modified protospacer adjacent motif (PAM) recognition (Kim et al., 2017b). Yet, the efficiency and specificity of base editors has not been demonstrated in human embryos. Here, we report that both base editor 3 (BE3) using nCas9 and SaKKH-BE3 using SaKKH-nCas9 can introduce single-nucleotide substitutions efficiently in human tripronuclear (3PN) zygotes.

We first used BE3 (rAPOBEC1-nCas9-UGI) to induce point mutations in human $\beta$-globin $(H B B)$, which associated with human diseases $\beta$-thalassemia (Fig. $1 \mathrm{~A}$ and $1 \mathrm{~B}$ ). We expected to introduce a premature stop codon in $H B B$ by G-to-A conversions at the target site. We carried out base editing in human $3 \mathrm{PN}$ zygotes by microinjection of $\mathrm{BE} 3$ mRNA and sgRNAs. The injected 3PN zygotes were cultured into 4 to 8-cell embryos and used for targeted-deep- sequencing analysis. Targeted point mutations were observed in 8 out of $19(42 \%)$ embryos at the target site in the $H B B$ gene, with mutation frequencies that ranged from $6 \%$ to $52 \%$ (Figs. 1C, 1D, 1K, and S1A). Targeted deep sequencing showed that 7 out of 8 embryos for $H B B$ base editing contained a nonsense mutation at the target site, generated by a single G-to-A conversion (Figs. 1D and S1A).

To broaden the genome-targeting scope of base editors, we used the recently reported SaKKH-BE3 that relaxes the variant's PAM requirement to NNNRRT (Fig. 1A). Targeted deep sequencing on the injected embryos revealed that 17 out of $17(100 \%)$ or 6 out of $9(67 \%)$ embryos carried targeted point mutations at the target site in the FANCF or DNMT3B gene, respectively (Fig. 1E-K). Note that we observed very low percentage $(<5 \%)$ of wild-type $(\mathrm{Wt})$ allele in 5 FANCF mutant embryos (FANCF-E2, E7, E9, E11, and E17) and no Wt allele in 3 FANCF mutant embryos (FANCF$E 13, E 14$, and E15), indicating high base-editing efficiencies in human 3PN embryos using SaKKH-BE3 (Figs. 1G, S1B, and S1C). Targeted deep sequencing showed that a C-to-T conversion was the major mutagenic pattern at all three target sites, with frequencies range from $78.8 \%$ to $98.5 \%$ (Fig. S2). C-to-A or C-to-G conversions were also observed in $1 \mathrm{HBB}(11 \%), 7$ FANCF $(70 \%)$, and 3 DNMT3B $(50 \%)$ mutant embryos (Figs. 1 and S1). We also found C-T conversion on the upstream or downstream of the sgRNA target site in 0 HBB (0\%), 10 FANCF (59\%), and 3 DNMT3B (50\%) mutant embryos (Fig. S2), consistent with previous studies (Kim et al., 2017a; Kim et al., 2017b; Komor et al., 2016). Using engineered base editors containing mutated cytidine deaminase domains, such as YE1-BE3, may narrow the width of the editing window (Kim et al., 2017b).

To avoid the PCR bias, we further examined 4- to 8-cell embryos with FANCF or DNMT3B base editing at the singlecell level. Single blastomeres of 4- to 8-cell embryos were isolated and picked up under the microscope for PCR amplification and Sanger sequencing. We found that 10 out of $10(100 \%)$ or 9 out of $9(100 \%)$ embryos carried targeted point mutations at the target site in the FANCF or DNMT3B gene, respectively (Figs. 1K, S3, and S4). Based on singlecell sequencing reads, $79 \%$ or $83 \%$ alleles carried targeted point mutations in the FANCF or DNMT3B (Fig. S5). Among these mutant embryos, two FANCF base-editing embryos 
A

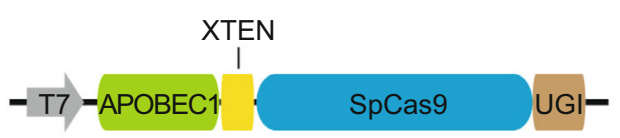

B

$B$

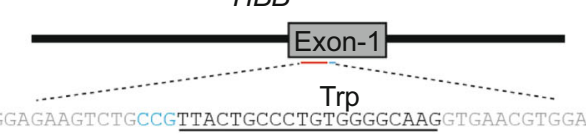

C

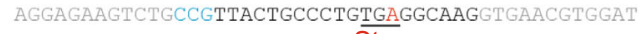

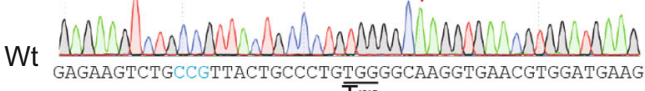
$\operatorname{Trp}$

E2

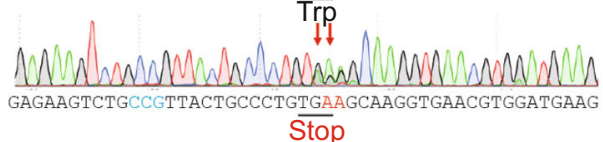

E

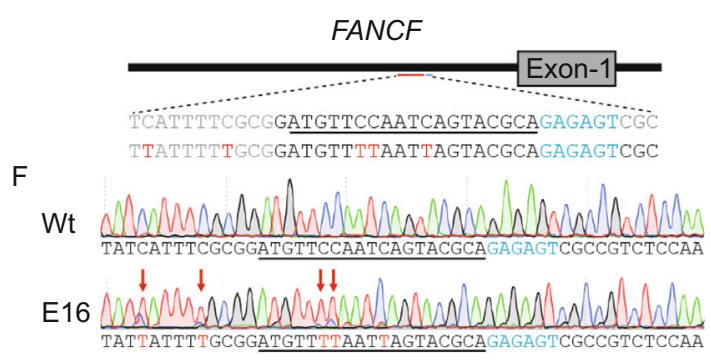

D

Wt AGGAGAAGTCTGCCGTTACTGCCCTGTGGGGAAGGTGAACGTGGAT 96

E1 AGGAGAAGTCTGCCGTTACTGCCCTGTGAGGCAAGGTGAACGTGGAT 52 (W16Stop) AGGAGAAGTCTGCCGTTACTGCCCTGTGGGGCAAGGTGAACGTGGAT 44 (Wt) AGGAGAAGTCTGCCGTTACTGCCCTGTGAAGCAAGGTGAACGTGGAT 20 (W16Stop, G17S)

E2 AGGAGAAGTCTGCCGTTACTGCCCTGTGGAGCAAGGTGAACGTGGAT 6 (G17S) AGGAGAAGTCTGCCGTTACTGCCCTGTGGGGCAAGGTGAACGTGGAT $71(\mathrm{Wt})$

E5 AGGAGAAGTCTGCCGTTACTGCCCTGTAAGGCAAGGTGAACGTGGAT 9 (W16Stop) AGGAGAAGTCTGCCGTTACTGCCCTGTGGGGCAAGGTGAACGTGGAT 87 (Wt) AgGAGAaGTCTGCCGTTACTGCCCTGTAaGGCAAGGTGAaCGTGGAT 16 (W16Stop)

E8 AGGAGAAGTCTGCCGTTACTGCCCTGTGAAGCAAGGTGAACGTGGAT 9 (W16Stop, G17S) AGGAGAAGTCTGCCGTTACTGCCCTGTGATGCAAGGTGAACGTGGAT 5 (W16Stop, G17S) AGGAGAAGTCTGCCGTTACTGCCCTGTGGGGCAAGGTGAACGTGGAT 60 (Wt)

E9 AGGAGAAGTCTGCCGTTACTGCCCTGTGAGGCAAGGTGAACGTGGAT 19 (W16Stop) G AGGAGAAGTCTGCCGTTACTGCCCTGTGGGGCAAGGTGAACGTGGAT 69 (Wt)

Wt tATCATtTCGCGGATGTTCCAATCAGTACGCAGAGAGTCGCCGTCTCCAA 92 TATCATTTCGCGGATGTTTTAATCAGTACGCAGAGAGTCGCCGTCTCCAA 68 $\begin{array}{ll}\text { TATCATTTCGCGGATGTTTAAATCAGTACGCAGAGAGTCGCCGTCTCCAA } & 23 \\ \text { TATCATTTCGCGGATGTTCCAATCAGTACGCAGAGAGTCGCCGTCTCCAA } & 2(\mathrm{Wt})\end{array}$ TATCATTTCGCGGATGTTCCAATCAGTACGCAGAGAGTCGCCGTCTCCAA 2 (V)

E13 TATCATTTCGCGGATGTTtAATCAGTACGCAGAGAgTCGCCGTCTCCAA 68 TATCATTTCGCGGATGTTTCAATTAGTACGCAGAGAGTCGCCGTCTCCAA 23

E15 tATCATtTCGCGGATGTTGCAATTAGTACGCAGAGAGTCGCCGTCTCCAA 89

E16 TATTATTTTGCGGATGTTTAATTAGTACGCAGAGAGTCGCCTCTCCAA 66 TATCATTTCGCGGATGTTCCAATCAGTACGCAGAGAGTCGCCGTCTCCAA $22(\mathrm{Wt})$

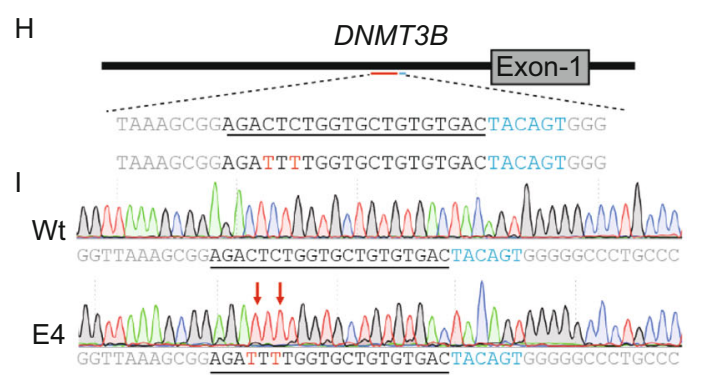
$\begin{array}{lll}\text { J PAM } & \text { Frequency (\%) }\end{array}$ GGAGGTGGGGGTTAAAGCGGAGATTITGGTGCTGTGTGACTACAGTGGGGG 41
E1 GGAGGTGGGGGTTAAAGCGGAGACTTTGGTGCTGTGTGACTACAGTGGGGG 33 GGAGGGGGGGGTTAAAGCGGAGATTTTGGTGCTGTGTGACTACAGTGGGGG 5 GGAGGTGGGGGTTAAAGCGGAGACTCTGGTGCTGTGTGACTACAGTGGGGG $18(\mathrm{Wt})$ GGAGGTGGGGGTTAAAGCGGAGATTTTGGTGCTGTGTGACTACAGTGGGGG 55
E4 GGAGGTGGGGGTTAAAGCGGAGACTTTGGTGCTGTGTGACTACAGTGGGGG 16 GGAGGTGGAGGTTAAAGCGGAGATTTTGGTGCTGTGTGACTACAGTGGGGG 8 GGAGGTGGGGGTTAAAGCGGAGACTCTGGTGCTGTGTGACTACAGTGGGGG $18(\mathrm{Wt})$ GGAGGTGGGGGTTAAAGCGGAGACTTTGGTGCTGTGTGACTACAGTGGGGG 43
E6 GGAGGTGGGGGTTAAAGCGGAGATTTTGGTGCTGTGTGACTACAGTGGGGG 26 GGAGGTGGGGTTAAAGCGGAGACTCTGGTGCTGTGTGACTACAGTGGGG $25(\mathrm{Wt})$

\begin{tabular}{|c|c|c|c|c|}
\hline Target gene & Base editors & $\begin{array}{c}\text { No. of } \\
\text { injected } \\
\text { embryos }\end{array}$ & $\begin{array}{l}\text { No. of two-cell } \\
\text { stage } \\
\text { embryos (\%) }\end{array}$ & $\begin{array}{c}\text { Mutant ratio (\%) } \\
\text { No. of mutants/total } \\
\text { embryos }\end{array}$ \\
\hline$H B B$ & BE3 & 22 & $19(86)$ & $8 / 19(42)^{a}$ \\
\hline FANCF & saKKH-BE3 & 18 & $17(94)$ & $17 / 17(100)^{a}$ \\
\hline DNMT3B & saKKH-BE3 & 9 & $9(100)$ & $6 / 9(67)^{a}$ \\
\hline FANCF & saKKH-BE3 & 10 & $10(100)$ & $10 / 10(100)^{b}$ \\
\hline DNMT3B & saKKH-BE3 & 9 & $9(100)$ & $9 / 9(100)^{b}$ \\
\hline
\end{tabular}

a PCR products of whole embryos were used for targeted deep sequencing.

${ }^{\mathrm{b}} \mathrm{PCR}$ products of single blastomere from divided embryos were used for Sanger sequencing.

(FANCF-E20, E24) and two DNMT3B base-editing embryos (DNMT3B-E11, E14) contained only targeted point mutations (Figs. S3 and S4). C-to-T conversion was the major mutagenic pattern, and C-to-A or C-to-G conversions were also observed in FANCF and DNMT3B mutant embryos (Fig. S5). Furthermore, compared with CRISPR/Cas9-mediated gene editing (Kang et al., 2016), although 7 out of 10 FANCF mutant embryos contained indels alleles (Fig. S3), the percentage of total DNA alleles with indels was very low $(13 \%$ for FANCF and $0 \%$ for DNMT3B) (Fig. S5D). Further optimizing base editors with inactive Cas9 mutant or Cpf1 mutant may reduce the indels to a lower level.

Finally, to assess base editors off-target effects, we performed whole genome sequencing (WGS) to identify SaKKH-BE3 off-target mutations in the three FANCF mutant embryos (FANCF-E28, E29, and E30) (Fig. S6). Of 1,187 possible off-target sites that differ from the on-target site by up to 5 mismatches, we observed just 1 potential off-target 
4 Figure 1. Base editing in human 3PN zygotes using BE3 and SaKKH-BE3. (A) Schematic diagram showing the structure of BE3 and SaKKH-BE3. XTEN, a linker; UGI, uracil glycosylase inhibitor. (B) The target sequence at the $H B B$ locus. The sgRNA sequencing and the PAM sequence are shown in black and blue, respectively. The nucleotide substituted by BE3mediated base editing is shown in red. (C) Sanger sequencing chromatograms of DNA from wild-type and HBB-E1 mutant embryo. The red arrow indicates the substituted nucleotide. The relevant codon identities at the target site are shown under the DNA sequence. (D) Alignments of mutant sequences from embryos with BE3-mediated editing at the $H B B$ locus. The target sequence is underlined. The substitutions and PAM site are shown in red and blue, respectively. The column on the right indicates the percent of relevant genotype in total sequencing reads. Wt, wild-type. (E) The target sequence at the FANCF locus. The nucleotide substituted by SaKKH-BE3-mediated base editing is shown in red. $(F)$ Sanger sequencing chromatograms of DNA from wild-type and FANCF-E16 mutant embryo. (G) Alignments of mutant sequences from embryos with SaKKH-BE3-mediated editing at the FANCF locus. $(\mathrm{H})$ The target sequence at the DNMT3B locus. The nucleotide substituted by SaKKH-BE3-mediated base editing is shown in red. (I) Sanger sequencing chromatograms of DNA from wild-type and DNMT3B-E4 mutant embryo. (J) Alignments of mutant sequences from embryos with SaKKH-BE3-mediated editing at the DNMT3B locus. $(\mathrm{K})$ Summary of base editing by BE3 and SaKKH-BE3 in human 3PN zygotes.

site in 1 out of 3 FANCF mutant embryos (Fig. S6). Taken together, these results indicate that BE3 did not induce significant off-target alterations in gene-edited human embryos.

In summary, our results show that microinjection of BE3 or SaKKH-BE3 mRNA resulted in efficient and precise base editing in human $3 \mathrm{PN}$ zygotes. These results demonstrate that base editors can be used for correcting genetic defects in human embryos in the future.

\section{FOOTNOTES}

This work was supported by grants from the National Natural Science Foundation of China (Grant No. 81671413), Shanghai Key Laboratory for Assisted Reproduction and Reproductive Genetic (17DZ2271100), National Research and Development Plan (2016YFC1000604), CAS Strategic Priority Research Program (XDB02050007, XDA01010409), the MoST863 Program (2015AA020307), NSFC grants (31522037), China Youth Thousand Talents Program (to H.Y.), and Break through project of Chinese Academy of Sciences.

C.Z. designed and performed experiments. M.Z. and Y.W. designed and performed human embryo injection. H.P. performed plasmid construction. Y.S. and Y.L. performed targeted-deep-sequencing analysis and off-target analysis. Y.S., Y. G., Y.D., Q.X., N. Y., W.Z., and Y.L. collected the human embryos. H.Y. designed experiments and wrote the manuscript. Z.C., H.Y., and W.L., supervised the project.
The authors declare no competing financial interests.

Changyang Zhou ${ }^{1,2}$, Meiling Zhang ${ }^{3}$, Yu Wei ${ }^{1}$, Yidi Sun ${ }^{2,5,6,7}$, Yun Sun ${ }^{3}$, Hong Pan ${ }^{1,8}$, Ning $\mathrm{Yao}^{3}$, Wanxia Zhong ${ }^{3}$, Yixue $\mathrm{Li}^{2,5,6,7}$, Weiping $\mathrm{Li}^{3 凶}$, Hui Yang ${ }^{1 凶}$, Zi-jiang Chen ${ }^{3,4 \bowtie}$

${ }^{1}$ Institute of Neuroscience, State Key Laboratory of Neuroscience, Key Laboratory of Primate Neurobiology, CAS Center for Excellence in Brain Science and Intelligence Technology, Shanghai Institutes for Biological Sciences, Chinese Academy of Sciences, Shanghai 200031, China

2 College of Life Sciences, University of Chinese Academy of Sciences, Beijing 100049, China

${ }^{3}$ Shanghai Key Laboratory for Assisted Reproduction and Reproductive Genetics, Center for Reproductive Medicine, Ren Ji Hospital, School of Medicine, Shanghai Jiao Tong University, Shanghai 200127, China

${ }^{4}$ Center for Reproductive Medicine, Shandong Provincial Hospital Affiliated to Shandong University, National Research Center for Assisted Reproductive Technology and Reproductive Genetics, The Key laboratory for Reproductive Endocrinology of Ministry of Education, Jinan 250021, China

${ }^{5}$ Key Lab of Computational Biology, CAS-MPG Partner Institute for Computational Biology, Shanghai Institutes for Biological Sciences, Chinese Academy of Sciences, Shanghai 200031, China

${ }^{6}$ Institute of Biomedical Sciences, Fudan University, Medical College, Shanghai 200032, China

7 Shanghai Center for Bioinformation Technology, Shanghai Industrial Technology Institute, Shanghai 201203, China

${ }^{8}$ State Key Laboratory for Conservation and Utilization of Subtropical Agro-Bioresource, Guangxi University, Nanning 530004, China

$\bowtie$ Correspondence: liweiping@renji.com (W. Li), huiyang@ion.ac.cn (H. Yang), chenzijiang@hotmail.com (Z. Chen)

\section{OPEN ACCESS}

This article is distributed under the terms of the Creative Commons Attribution 4.0 International License (http://creativecommons.org/ licenses/by/4.0/), which permits unrestricted use, distribution, and reproduction in any medium, provided you give appropriate credit to the original author(s) and the source, provide a link to the Creative Commons license, and indicate if changes were made.

\section{REFERENCES}

Kang X, He W, Huang Y, Yu Q, Chen Y, Gao X, Sun X, Fan Y (2016) Introducing precise genetic modifications into human 3PN embryos by CRISPR/Cas-mediated genome editing. J Assist Reprod Genet 33:581-588

Kim K, Ryu SM, Kim ST, Baek G, Kim D, Lim K, Chung E, Kim S, Kim JS (2017a) Highly efficient RNA-guided base editing in mouse embryos. Nat Biotechnol 35:435-437 
Kim YB, Komor AC, Levy JM, Packer MS, Zhao KT, Liu DR (2017b) Increasing the genome-targeting scope and precision of base editing with engineered Cas9-cytidine deaminase fusions. Nat Biotechnol 35:371-376

Komor AC, Kim YB, Packer MS, Zuris JA, Liu DR (2016) Programmable editing of a target base in genomic DNA without double-stranded DNA cleavage. Nature 533:420-424

Liang P, Sun H, Sun Y, Zhang X, Xie X, Zhang J, Zhang Z, Chen Y, Ding $C$, Xiong $Y$ et al (2017) Effective gene editing by high-fidelity base editor 2 in mouse zygotes. Protein Cell 8(8):601-611

Liang $P, X u Y$, Zhang $X$, Ding $C$, Huang R, Zhang Z, Lv J, Xie X, Chen Y, Li Y et al (2015) CRISPR/Cas9-mediated gene editing in human tripronuclear zygotes. Protein Cell 6:363-372

Long C, McAnally JR, Shelton JM, Mireault AA, Bassel-Duby R, Olson EN (2014) Prevention of muscular dystrophy in mice by CRISPR/Cas9-mediated editing of germline DNA. Science 345:1184-1188

Ma Y, Zhang J, Yin W, Zhang Z, Song Y, Chang X (2016) Targeted AID-mediated mutagenesis (TAM) enables efficient genomic diversification in mammalian cells. Nat Methods 13:1029-1035

Nishida K, Arazoe T, Yachie N, Banno S, Kakimoto M, Tabata M, Mochizuki M, Miyabe A, Araki M, Hara KY et al (2016) Targeted nucleotide editing using hybrid prokaryotic and vertebrate adaptive immune systems. Science 353:aaf8729

Tang L, Zeng Y, Du H, Gong M, Peng J, Zhang B, Lei M, Zhao F, Wang W, Li X et al (2017) CRISPR/Cas9-mediated gene editing in human zygotes using Cas9 protein. Mol Genet Genom 292:525-533

Wang H, Yang H, Shivalila CS, Dawlaty MM, Cheng AW, Zhang F, Jaenisch R (2013) One-step generation of mice carrying mutations in multiple genes by CRISPR/Cas-mediated genome engineering. Cell 153:910-918

Wu Y, Liang D, Wang Y, Bai M, Tang W, Bao S, Yan Z, Li D, Li J (2013) Correction of a genetic disease in mouse via use of CRISPR-Cas9. Cell Stem Cell 13:659-662

Yang H, Wang H, Shivalila CS, Cheng AW, Shi L, Jaenisch R (2013) One-step generation of mice carrying reporter and conditional alleles by CRISPR/Cas-mediated genome engineering. Cell 154:1370-1379

Zong Y, Wang Y, Li C, Zhang R, Chen K, Ran Y, Qiu JL, Wang D, Gao C (2017) Precise base editing in rice, wheat and maize with a Cas9-cytidine deaminase fusion. Nat Biotechnol 35:438-440
Changyang Zhou, Meiling Zhang, Yu Wei, and Yidi Sun have contributed equally to this work.

Electronic supplementary material The online version of this article (doi:10.1007/s13238-017-0459-6) contains supplementary material, which is available to authorized users. 